\title{
Transtorno de Personalidade Borderline e sua relação com os comportamentos autodestrutivos e suicídio
}

\author{
Borderline Personality Disorder and how it connects to self-destructive behaviors and \\ suicide
}
Trastorno Limíte de la Personalidad y su relación con las conductas autodestructivas y el suicidio

Caroline Silva de Araujo Lima ${ }^{1 *}$, Ana Luiza Rodrigues de Almeida², Bruna Ellen Muniz de Souza ${ }^{3}$, Cátia Ribeiro de Sá Rodrigues ${ }^{4}$, Izabella Caser Lopes de Faria ${ }^{5}$, Júlia Mariano Leiael Nascimento ${ }^{6}$ Mariah Vicari Bolognani ${ }^{7}$, Samanttha Cristina da Silva Chaves ${ }^{8}$, Gustavo Fonseca Genelhu Soares ${ }^{9}$.

\section{RESUMO}

Objetivo: Descrever e revisar os conhecimentos acerca da relação entre Transtorno de Personalidade Borderline, comportamentos autodestrutivos e o suicídio. Revisão bibliográfica: Os transtornos de personalidade são importantes fatores de risco para o suicídio, sendo a maioria dos comportamentos suicidas atribuíveis ao tipo Borderline. O comportamento suicida é relacionado, também, à gravidade da doença e ao baixo nível socioeconômico. O desenvolvimento e o curso do transtorno são variáveis, e o mais comum é que ocorram instabilidade nas relações interpessoais e na autoimagem e descontrole afetivo, com impulsividade intensa. Os prejuízos causados pelo transtorno na vida dos acometidos são, portanto, significativos e, apesar de poder haver alguma melhora com o aumento da idade e com abordagens terapêuticas, não há cura. Considerações finais: Por meio da análise dos impactos negativos, causas, desenvolvimento e consequências do Transtorno de Personalidade Borderline, o presente estudo reforçou o aumento do risco do suicídio e fenômenos autodestrutivos nos acometidos.

Palavras-chave: Transtorno de Personalidade Borderline, Suicídio, Psicopatologia.

\begin{abstract}
Objective: To describe and review the knowledge about the relationship between Borderline Personality Disorder, self-destructive behavior and suicide. Bibliographic review: Personality disorders are important risk factors for suicide, most suicidal behaviors being attributable to the Borderline type. Suicidal behavior is also related to the severity of the disease and low socioeconomic level. The development and course of the disorder are variable, and the most common pattern is the occurrence of instability in interpersonal relationships and self-image and affective lack of control, with intense impulsivity. The damage caused by the disorder in the lives of those affected is therefore significant and, although there may be some improvement with increasing age and therapeutic approaches, there is no cure. Final considerations: Through the analysis of the negative impacts, causes, development and consequences of the Borderline Personality Disorder, this study has reinforced the increased risk of suicide and self-destructive phenomena in those affected.
\end{abstract}

Keywords: Borderline Personality Disorder, Suicide, Psychopathology.

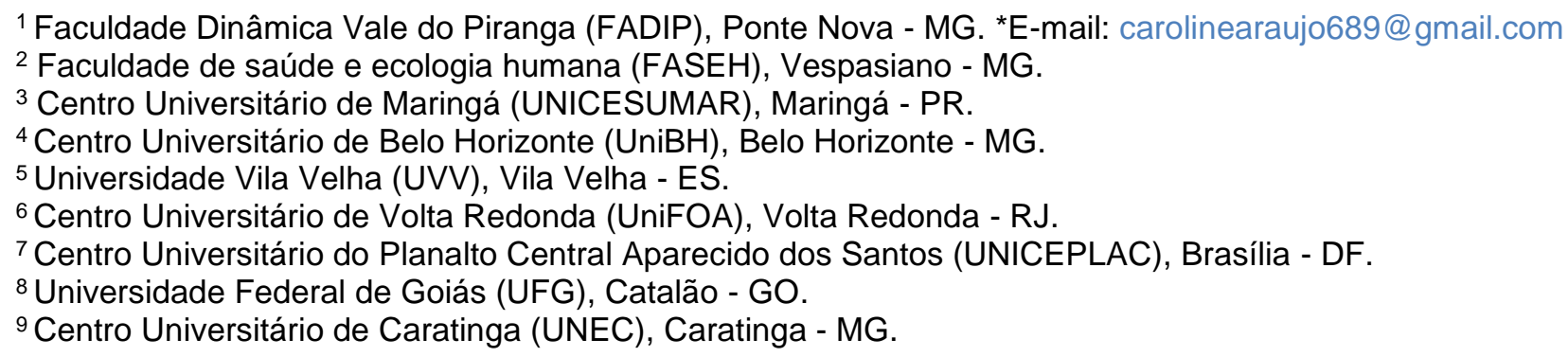




\section{RESUMEN}

Objetivo: Describir y repasa el conocimiento sobre la relación entre el Trastorno de Personalidad Limítrofe, el comportamiento autodestructivo y el suicidio. Revisión bibliográfica: Los trastornos de personalidad son factores de riesgo importantes para el suicidio, y la mayoría de las conductas suicidas son atribuibles al tipo Borderline. El comportamiento suicida también está relacionado con la gravedad de la enfermedad y el bajo nivel socioeconómico. El trastorno tiene un desarrollo y un curso variables, y la pauta más común es la de episodios graves de falta de control afectivo e inestabilidad en las relaciones interpersonales, la imagen de sí mismo y las relaciones afectivas, con una marcada impulsividad. Por consiguiente, el daño causado por el trastorno en la vida de los afectados es importante $y$, aunque puede haber cierta mejora con el aumento de la edad y los enfoques terapéuticos, no hay cura. Consideraciones finales: Mediante el análisis de los efectos negativos, las causas, el desarrollo y las consecuencias del trastorno de la personalidad limítrofe, el presente estudio reforzó el aumento del riesgo de suicidio y de fenómenos autodestructivos en los afectados.

Palabras clave: Trastorno de Personalidad Limítrofe, Suicidio, Psicopatología.

\section{INTRODUÇÃO}

Um dos mais complexos fenômenos psíquicos, conhecido como Transtorno de Personalidade Borderline (TPB), vêm sendo cada vez mais estudado e alimentado cientificamente na literatura, visto que sua ocorrência influencia muito no dia a dia de seus portadores, seja em fenômenos individuais ou de coletividade, como no relacionamento com outras pessoas (YEN S, et al., 2017).

Tal fenômeno resulta em impactos negativos no cotidiano e em comportamento auto prejudicial e nocivo para os indivíduos acometidos e, assim, elevam-se as taxas de suicídio pelo alto grau de comprometimento psíquico (SOLOFF PH e CHIAPPETTA L, 2017).

As crises do TPB não apresentam causas definidas, mas, geralmente, acontecem após um evento marcante, como divórcio ou morte. A crise, acompanhada por instabilidade de relacionamento interpessoal, de identidade, de afeto e de impulsividade, acarreta danos emocionais incuráveis aos afetados, como o término de relacionamentos com pessoas queridas, traumas psicológicos dificilmente esquecidos e perda da autocontrolabilidade (APA, 2014).

Embora apresente desenvolvimento e curso variáveis, as consequências do transtorno levam à vulnerabilidade e ao sofrimento emocional, bem como dificuldades em se relacionar, que acarretam um maior risco de automutilação e tentativa de suicídio a esses pacientes. Um estudo realizado por Goodman $\mathrm{M}$, et al. (2017) com 394 pacientes internados com Transtorno de Personalidade Limítrofe, que objetivava determinar a frequência com que pacientes internados realizavam atos fisicamente autodestrutivos, ou seja, automutilação e tentativas de suicídio, encontrou incidência acima de $90 \%$ de relatos de automutilação e índices superiores a $75 \%$ de tentativas de autoextermínio. Embora o estudo tenha sido realizado somente com pacientes internados e em tratamento, as altas taxas são um resultado alarmante (MAMMEN O, et al., 2020).

Outro estudo destacou, ainda, o importante papel da família como rede de apoio a este paciente, pois ajuda a desenvolver um entrelaçamento com famílias em torno de uma compreensão conjunta acerca dos resultados no suicídio crônico. O estudo mostrou que a maioria dos pacientes acaba tendo resultados melhores quando há o suporte familiar, porém, o risco de tentativas de autoextermínio permanece. Isso permite que os pacientes e as famílias façam uma escolha mais assertiva e munida de informações sobre 0 tratamento, o que pode reduzir o risco na minoria de casos em que o paciente comete suicídio (MAMMEN O, et al., 2020).

A associação entre TPB e a automutilação vem crescendo, assim como sua relação com o comportamento violento e relatos de experiências semelhantes às psicóticas. Tais fatos demonstram a forte ligação entre questões sociopsicológicas familiares, eventos traumáticos e negligência como fatores desencadeantes do desenvolvimento comportamental do paciente (REISING K, et al., 2019; SENGUTTA M, et al., 2018). 
Recentemente, indivíduos com idade inferior a dezoito anos também foram incluídos no diagnóstico de Transtorno de Personalidade Borderline e, assim, esse transtorno é cada vez mais reconhecido como uma doença legítima e que precisa de tratamento e intervenção precoces. O TPB do adolescente agora está incluído no DSM-5, e, ainda, especifica um subtipo em que traços de afetividade negativos estão presentes (APA, 2014).

Diante do exposto, o estudo tem como orientação a seguinte pergunta: "Como os impactos negativos do TB influenciam no dia a dia do paciente?". Tendo em vista, que as recentes pesquisas demonstram os impactos negativos do transtorno de personalidade limítrofe no dia a dia do paciente, bem como sua associação com o suicídio, afetando assim, diversas áreas da vida. Onde, em casos mais graves, a integridade física também pode ser colocada em risco. Dessa forma, este trabalho tem como objetivo identificar e revisar essa relação e as consequências mais variadas que tal transtorno traz hodiernamente ao indivíduo.

\section{REVISÃO BIBLIOGRÁFICA}

O TPB é também conhecido como Transtorno de Personalidade Limítrofe. A expressão Borderline referese a algo que está na fronteira, no limite. O TPB é caracterizado por um padrão difuso de instabilidade nas relações interpessoais, na autoimagem e nas relações afetivas, com acentuada impulsividade em diferentes contextos, acarretando dificuldades de convívio e instabilidade social, seja para o paciente, seja para as pessoas a seu redor, o que causa sofrimento e prejuízos em diversos âmbitos (APA, 2014).

O TPB tem uma prevalência aproximada de $1,6 \%$ na população, mas pode chegar a 5,9\%. Na atenção primária, essa prevalência é estimada em $6 \%$, e alcança cerca de 10\% entre pacientes de ambulatórios de saúde mental e $20 \%$ entre pacientes internados devido a doenças psiquiátricas. A prevalência do transtorno comumente é menor em faixas etárias mais altas (APA, 2014). Pressupõe-se que o transtorno seja duas vezes mais comum no sexo feminino do que no masculino. Além disso, indivíduos com TPB frequentemente têm antecedentes familiares de transtorno depressivo maior, transtornos por uso de álcool e de abuso de substância, em parentes de primeiro grau. Pessoas com transtorno da personalidade borderline também são mais propensas a apresentarem algum transtorno do humor (KAPLAN HI e SADOCK BJ, 2014).

Esses indivíduos costumam apresentar um padrão difuso de instabilidade das relações interpessoais, da autoimagem e dos afetos e de impulsividade acentuada. Para tanto, o Manual Diagnóstico e Estatístico de Transtornos Mentais traduz o diagnóstico na ocorrência de pelo menos 5 dos seguintes fatores, podendo ser estabelecido a partir do início da idade adulta, facilitando o diagnóstico precoce e o tratamento. São eles: (i) Esforços desesperados para evitar abandono real ou imaginado; (ii) Um padrão de relacionamentos interpessoais instáveis e intensos; (iii) Perturbação da identidade; (iv) Impulsividade em pelo menos duas áreas potencialmente autodestrutivas; (v) ameaças suicidas ou de comportamento automutilante; (vi) Instabilidade afetiva devido a uma acentuada reatividade de humor; (vii) Sentimentos crônicos de vazio; (viii) Raiva intensa e inapropriada ou dificuldade em controlá-la; (ix) Ideação paranóide transitória associada a estresse ou sintomas dissociativos intenso (APA, 2014). Diante desses fatores, é importante ressaltar que tal descrição facilitou a compreensão dos sinais e sintomas, tanto dos profissionais da saúde quanto dos familiares e pessoas próximas.

\section{Impactos Negativos do Transtorno}

O Transtorno de Personalidade (TP) é fator de risco importante para suicídio, sendo a maioria dos comportamentos suicidas atribuíveis àqueles com TPB. Segundo a Organização Mundial da Saúde (OMS), de 15.629 casos computados de suicídio, $11,6 \%$ e 35,8\% são efetuados por indivíduos com algum transtorno de personalidade e de humor, respectivamente (BRASIL, 2021). Estes traços de comportamento suicida estão relacionados também à gravidade da doença e ao baixo nível socioeconômico, além de instabilidade afetiva e comportamento agressivo, que são característicos de temperamento associados a comportamento suicida (SOLOFF PH e CHIAPPETTA L, 2017). 
Em relação ao comportamento, a agressividade, por vezes, é caracterizada como de difícil contenção, devido a essas relações de ódio do paciente borderline serem muito intensas. Um elemento importante acerca da agressividade é aquele voltado para o próprio sujeito e que aponta o alto risco de suicídio, além de se relacionar às ameaças frequentes de autodestruição e aos comportamentos autolesivos (SANTOS GG e NETO GARM, 2018).

Em um estudo realizado na Holanda, foram analisados 71.707 pacientes com idade entre 18 e 65 anos em admissão psiquiátrica voluntária ou involuntária. Os dados foram obtidos em três áreas da Holanda de 2007 a 2016, sendo alguns pacientes pós tentativa de suicídio ou com pensamentos suicida atendidos pelo serviço de emergência psiquiátrica. Neste estudo, foram usados dados de um arquivo eletrônico do paciente, um sistema de suporte clínico que compreende informações sobre variáveis sociodemográficas, sintomas psiquiátricos, diagnósticos psiquiátricos e dados ambientais (VAN VEEN M, 2019).

Os pacientes foram atendidos pelo psiquiatra juntamente com uma enfermeira, ou um médico ou residente em psiquiatria, supervisionado por um psiquiatra, mas às vezes também a pedido da polícia ou de um serviço de emergência de um hospital geral. Os ferimentos causados por tentativas de suicídio levaram a 93,8 atendimentos em departamentos de emergência e 56,3 admissões em hospitais gerais por 100.000 habitantes em 2015. Independentemente do nível de risco de suicídio, os pacientes suicidas com diagnóstico de transtorno de personalidade eram menos propensos a serem admitidos voluntariamente do que aqueles sem esse diagnóstico (taxa de admissão de 0,37 versus 0,46 , respectivamente). No entanto, quando o nível de risco de suicídio era moderado ou alto, aqueles com transtorno de personalidade internados involuntariamente tinham a mesma probabilidade de admissão involuntária que aqueles sem esse transtorno (VAN VEEN M, 2019).

Verificou-se, com isso, a importância de avaliar individualmente cada caso dentre os pacientes admitidos. Dessa forma, o objetivo principal do tratamento é avaliar a presença de um transtorno mental, estimar o risco para si ou para terceiros e iniciar uma intervenção, a fim de encontrar a melhor alternativa de abordagem terapêutica para o paciente. A internação hospitalar é uma questão de debate profissional, visto que especialistas argumentam que a internação involuntária acarreta na perda de autonomia para esse paciente, aumentando, assim, o risco de suicídio (VAN VEEN M, 2019).

\section{Causas e Desenvolvimento do Transtorno}

Conforme é descrito por National Institute of Mental Health (NIMH), as causas do TPB não são completamente claras, mas pesquisas sugerem que a história familiar, mudanças estruturais e funcionais no cérebro, fatores sociais, culturais e ambientais, podem influenciar o risco de um indivíduo desenvolver o transtorno. No entanto, o fato de o risco ser aumentado, não implica, necessariamente, no desenvolvimento do TPB. Da mesma forma, indivíduos que não apresentam tais fatores de risco podem desenvolver a patologia em algum período da vida (NIMH, 2020)

Em comparação com a população geral, o TPB é apresentado mais frequentemente em indivíduos que tenham parentes biológicos de primeiro grau com o transtorno (APA, 2014). Em relação às mudanças estruturais e funcionais do cérebro, é possível destacar mudanças especialmente nas áreas que controlam impulso, regulam emoções e uma possível disfunção em algumas substâncias químicas como a serotonina. Dentre os fatores sociais, culturais e ambientais, é importante destacar a influência de eventos traumáticos como abuso físico, sexual e emocional; abandono; algum acontecimento marcante na infância, como a separação dos pais; relacionamentos instáveis; situações hostis e invalidações emocionais (NIMH, 2020).

O desenvolvimento e o curso do transtorno na vida de um indivíduo são variáveis. O comportamento mais frequente consiste na instabilidade de longa duração na fase inicial da vida adulta, sobressaindo eventos de falta de controle afetivo e impulsivo considerados graves (APA, 2014). Dado que há uma relação entre altos índices de suicídio e o TPB entre adultos jovens e de automutilação, o prejuízo causado pela doença na vida de um indivíduo é significativo (NIMH, 2020). Além disso, a automutilação é um comportamento de autodestruição oriundo de um desejo de se punir, que pode ser inconsciente e afetar consideravelmente um indivíduo (VIEIRA MG, et al., 2016). 
No entanto, indivíduos que se comprometem com as ações terapêuticas propostas, a melhora de comportamento em relação a impulsividade, emoções e relacionamentos intensos é notória. Além disso, é notável que na faixa dos 30 até os 50 anos de idade, a maioria dos indivíduos com o transtorno atinge uma condição constante nos seus relacionamentos e no âmbito profissional. Alguns estudos de seguimento de indivíduos identificados por intermédio de clínicas de saúde mental inferem que, após cerca de uma década, pelo menos metade dos pacientes não apresentam mais um padrão comportamental que atenda aos critérios necessários para o Transtorno de Personalidade Borderline (APA, 2014).

\section{Consequências do Transtorno}

O TPB é um quadro complexo, que apresenta consequências que afetam tanto a vida dos pacientes, quanto o convívio social e familiar (MUGARTE IB, 2014). De acordo com os critérios do Diagnostic and Statistical Manual of Mental Disorders 5 (DSM-5, 2014), algumas características relacionadas ao TPB são: a instabilidade afetiva, a necessidade de evitar o abandono real ou imaginário, a impulsividade, o sentimento de vazio e a ideação suicida (APA, 2014). Esses sentimentos podem durar minutos ou poucas horas e, mais raramente, duram alguns dias, alternando entre momentos de estabilidade e descontrole (CERUTTI PS e DUARTE TC, 2016).

Devido aos sentimentos crônicos de vazio, é comum que o sujeito se submeta a relações de abusos, para pertencer a um determinado grupo ou pessoa, buscando evitar o abandono. Dessa forma, a grande dependência e idealização acerca dos relacionamentos interpessoais, gera grande dificuldade em controlar a ansiedade frente às frustrações, desencadeando, assim, sintomas depressivos (MATIOLI MR, et al., 2014). Além disso, a necessidade de alívio do sofrimento, muitas vezes, se dá através de comportamentos autodestrutivos, os quais podem variar entre leves arranhões até comportamentos suicidas, buscando deslocar a atenção da dor emocional para uma lesão palpável (MACEDO BBD, et al., 2017).

Os comportamentos autodestrutivos são uma forte consequência do transtorno de personalidade Borderline. Estes caracterizam-se como a prática de se cortar em busca de alívio para uma dor psíquica intensa que vem de um desejo de se punir e apresenta grande impacto na vida do indivíduo (VIEIRA MG, et al., 2016). Confusões mentais causadas pelo transtorno é, na maioria das vezes, a explicação para as ações de automutilação e busca de alívio do indivíduo com o seu psíquico (FINKLER DC, et al., 2017).

\section{Tratamento do Transtorno e prevenção do suicídio}

A abordagem aos pacientes com Borderline, quando precoce e de maneira adequada, com apoio de uma equipe multidisciplinar incluindo médicos, psicólogos e psiquiatras, com a colaboração familiar e de forma constante, contribui para seu controle, evitando a automutilação, uma consequência fatal que é o suicídio, ato de tirar a própria vida, objetivando um alívio total para os sentimentos psíquicos excessivos presentes no TPB. Dessa forma, a associação do tratamento farmacológico com o psicológico é necessária o quanto antes. Várias intervenções psicológicas se mostraram eficazes no tratamento do Borderline, dentre elas a Terapia Cognitivo-comportamental (TCC), que é apontada como a mais eficaz (MARTINS L e DOTA F, 2017). A TCC, além de melhoria clínica dos pacientes, consegue mantê-los por mais tempo no tratamento, aliviando sintomas de ansiedade e depressão (POWELL VB, et al., 2008).

No âmbito terapêutico, há a Terapia Comportamental Dialética (TCD) que é uma abordagem eficiente no tratamento do Transtorno de Personalidade Borderline, pois coloca a relação terapêutica como um de seus pilares devido ao uso da relação como forma de persuasão e modificação de comportamentos desadaptativos. Essa terapia é um tipo de Terapia Cognitivo-Comportamental que emprega estratégias baseadas em técnicas de aceitação, métodos e conceitos da ciência comportamental, algumas práticas incorporadas da prática zen budista e outros métodos contemplativos, como o mindfulness. Com isso, visa-se a validação e aceitação dos pacientes como eles são, auxiliando no processo de mudança do que eles se tornaram no momento presente (CAVALHEIRO CV e MELO WV, 2016).

Além do tratamento proposto pela terapia individual, há a terapia em grupo que concede a experiência de transpor sentimentos e atitudes, a resolução de possíveis bloqueios interpessoais e proporciona uma 
identificação e auxílio ao núcleo da personalidade do indivíduo, podendo atuar concomitantemente com a terapia individual. Com isso, o paciente tende a não abandonar prematuramente a terapia. É importante ressaltar que intervenções familiares e a observação do convívio do paciente em diversos núcleos sociais pode ser necessária para que o tratamento seja promissor, identificando o papel da família na origem e na progressão do transtorno e para que haja a preservação da sintomatologia estável do paciente. Assim, é possível contribuir positivamente para uma formação sólida de uma rede de apoio ao paciente Borderline (DAL'PZOL A, et al., 2003).

Em vista disso, a psicoterapia é um dos tratamentos fundamentais para o transtorno, mas quando combinado com a farmacologia, pode agir em conjunto levando melhores resultados aos pacientes (FINKLER DC, et al., 2017). Fármacos como antipsicóticos típicos, incluindo a trifluoperazina, podem melhorar sintomas de ansiedade, colaborando grandemente para uma melhora no quadro de pacientes com o transtorno de Borderline. Além do mais, estabilizadores de humor e inibidores seletivos da receptação da serotonina podem colaborar para a diminuição dos quadros constantes de mudança de humor e também de pensamentos pessimistas, que geralmente desenvolvem para a automutilação e o suicídio. É importante levar em conta os cuidados com os portadores do transtorno, uma vez que é um dos mais prevalentes no âmbito clínico e pode acarretar em muitos danos fatais (FINKLER DC, et al., 2017).

A partir de uma abordagem interdisciplinar é possível obter uma melhora integral desses pacientes. Observou-se com essa abordagem integral uma condição constante da apresentação clínica psiquiátrica e, principalmente, a obtenção de um exercício pleno e adequado de atividades cotidianas.É importante ressaltar que a ideia de estabilidade que um tratamento bem-organizado transmite ao paciente Borderline é de confiança e acolhimento, pois o indivíduo percebe que pode contar com uma equipe de profissionais coesa, bem como um serviço apto a corresponder às suas necessidades (DAL'PZOL A, et al., 2003).

Em relação a um possível comportamento suicida, é preciso atenção a frases de alerta como "os outros serão mais felizes sem mim" ou "eu não aguento mais", entre outras. A ajuda que deve ser oferecida consiste em, primeiramente, estabelecer um contato inicial e encontrar um local adequado, onde haja uma tranquilidade e privacidade razoáveis. Em seguida, é preciso reservar o tempo necessário e estar disponível emocionalmente para dar atenção a esses indivíduos com uma escuta ativa, transmitindo mensagens verbais e não verbais de aceitação e respeito aos sentimentos e valores da pessoa (BRASIL, 2021).

Dentro desse cenário, caso o indivíduo tenha um plano definido e os meios para realizar o suicídio, planeja fazê-lo prontamente e muitas vezes já tomou algumas providências prévias e parece estar se despedindo é preciso que ele não fique sozinho, gentilmente falar com a pessoa e remover os meios para que ela não cometa suicídio. Além disso, o tratamento psicoterápico ajuda no processo de mudança de comportamento, por mais que existam diversos desafios como o de admitir que tem tal transtorno e que necessita de ajuda. É válido também, informar a família e reafirmar que a rede de apoio é importante, pois trata-se de uma situação de emergência e faz-se urgente a medida de entrar em contato com um profissional de saúde mental ou do serviço de emergência mais próximo (BRASIL, 2021).

\section{CONSIDERAÇÕES FINAIS}

Diante do exposto, este estudo reforçou a associação entre o Transtorno de Personalidade Borderline, comportamentos autodestrutivos e suicídio, evidenciando a urgência em amenizar o sofrimento emocional e os comportamentos prejudiciais dos acometidos por este Transtorno. Para isso, são necessários estudos mais aprofundados acerca de novas intervenções psicoterápicas e farmacológicas que envolvam o indivíduo de forma integral, a fim de encontrar a melhor alternativa de abordagem terapêutica para o paciente, além de averiguar a eficácia das já existentes. Além disso, um tratamento adequado e organizado deve incluir manejo do risco de suicídio e medidas adjuntas para os sintomas de TPB, transmitindo segurança e confiança para que o paciente possa se sentir acolhido durante o tratamento. 


\section{REFERÊNCIAS}

1. ANDREWES HE, et al. Relationships between the frequency and severity of non-suicidal self-injury and suicide attempts in youth with borderline personality disorder. Early Intervention In Psychiatry 2017; 13(2): $194-201$.

2. APA. Manual diagnóstico e estatístico de transtornos mentais (DSM-5). American Psychiatric Association. 2014. Disponível em: periodicos.pucpr.br. Acessado em: 17 de novembro de 2020.

3. ARAÚJO JFB, et al. O corpo na dor: automutilação, masoquismo e pulsão. Estilos da Clínica, 2016; 21(2), 497-515.

4. BRASIL. Ministério da Saúde. Prevenção do suicídio: manual dirigido a profissionais das equipes de saúde mental. Disponível em: https://www.cvv.org.br/wpcontent/uploads/2017/05/manual_prevencao_suicidio_profissionais_saude.pdf. Acessado em: 21 de março de 2021.

5. CAVALHEIRO CV, MELO WV. Relação terapêutica com pacientes borderlines na terapia comportamental Dialética. Psicologia em Revista, 2016; 22(3): 579-595.

6. CERUTTI PS, DUARTE TC. Transtorno da personalidade borderline sob a perspectiva da terapia comportamental dialética. Revista Psicologia em Foco 2016; 8(12): 67-81.

7. DAL'PZOL A, et al. Programa de abordagem interdisciplinar no tratamento do transtorno de personalidade borderline - relato da experiência no ambulatório Melanie Klein do Hospital Psiquiátrico São Pedro. Revista de Psiquiatria Rio Grande do Sul, 2003; 25(1): 42-51.

8. FINKLER DC, et al. Transtorno de personalidade borderline: Estudos brasileiros e considerações sobre a DBT. Revista Brasileira de Terapia Comportamental e Cognitiva, 2017; 19(3): 274-292.

9. GOODMAN M, et al. Suicide Attempts and Self-Injurious Behaviors in Adolescent and Adult Patients with Borderline Personality Disorder. Personal Ment Health, 2017; 11(3): 157-163

10. KAPLAN \& SADOCK. Compêndio de Psiquiatria. 11aㅡ ed. Editora Artmed. 2014.

11. REISING K, et al. Childhood risk factors for personality disorder symptoms related to violence. Elsevier, 2019; 49:114.

12. SENGUTTA M, et al. The mediating role of borderline personality features in the relationship between childhood trauma and psychotic-like experiences in a sample of help-seeking non-psychotic adolescents and young adults. Elsevier, 2019; 56: 54-90.

13. MACEDO BBD, et al. Consequências da personalidade borderline na adesão ao tratamento de dores crônicas. Revista de Humanidades, 2017; 32(2): 193-203.

14. MARTINS L, DOTA F. Transtorno de personalidade borderline: a intervenção da terapia cognitivo comportamental. Revista Científica Eletrônica de Psicologia, 2017; 29(1): 3-26.

15. MATIOLI MR, et al. O transtorno de personalidade borderline a partir da visão de psicólogas com formação em Psicanálise. Saúde Transform. Soc., 2014; 5(1): 50-57.

16. MAMMEN O, et al. Communicating suicide risk to families of chronically suicidal borderline personality disorder patients to mitigate malpractice risk. Elsevier, 2020; 67: 51-57.

17. MUGARTE IB. Borderline. Singular Sociais e Humanidades, 2019; 1(1): 13-20.

18. NIMH.National Institute of Mental Health. Borderline Personality Disorders Theory and Management. Disponível em: https://www.nimh.nih.gov/health/publications/borderline-personality-disorder/borderlinepersonalitydis-508-qf-174928_156499.pdf. Acessado em: 15 de novembro de 2020.

19. SHIRLEY Y, et al. Borderline personality disorder in suicidal adolescents. Personality and Mental Health, 2013; 7: 89101.

20. SOLOFF PH, CHIAPPETTA L. Suicidal Behavior and Psychosocial outcome in Borderline Personality Disorder at 8year follow-up. Journal of Personality Disorders, 2017; 31:1-16.

21. VAN VEEN $M$, et al. Suicide risk, personality disorder and hospital admission after assessment by psychiatric emergency services. BMC psychiatry, 2019; 19(1): 157, 2019.

22. VIEIRA MG, et al. Self-mutilation: pain intensity, triggering and rewarding factors. Revista Dor, 2016; 17(4): 257-260.

23. WHO. World Health Organization. Mental Health. Prevention of Suicidal Behaviours: A Task for All. Disponível em: http://www.who. int/mental_health/prevention/suicide/background. Acessado em: 10 de novembro de 2020.

24. SANTOS GG, NETO GARM. Patients, problems and borders: psychoanalysis and borderline cases. Psicologia USP, 2018; 29(2): 285-293.

25. YEN S, et al. Borderline personality disorder in suicidal adolescents. Personality and mental health, $2013 ; 7: 89-101$.

26. POWELL VB, et al. Terapia cognitivo-comportamental da depressão. Rev. Bras. Psiquiatr., 2008; 30(2): 1-8. 